\title{
Persistent left superior vena cava: implications during central venous cannulation
}

\author{
Andrew Verniquet, MD • Rafid Kakel, MD
}

Received: 21 June 2011 / Accepted: 18 October 2011/Published online: 1 November 2011

(C) Canadian Anesthesiologists' Society 2011

\section{To the Editor,}

We read with interest the article by Commandeur et al. entitled "Ultrasound-guided cannulation of the left subclavian vein in a case of persistent left superior vena cava (PLSVC)." 1 The authors did not mention in their article the possibility of having cannulated the left pericardiophrenic vein, which can be mistaken for the PLSVC on an anterior chest radiograph except that it follows the left superior mediastinal border and then curves laterally along the left heart border (whereas a PLSVC curves medially). ${ }^{2}$

Normally, a PLSVC drains into the right atrium. Drainage into the left atrium (as was stated in the report) is rare, creating a right-to-left shunt with associated risks of hypoxemia and systemic embolization. Usually, a PLSVC coexists with a right superior vena cava (RSVC); and the two may or may not be connected by a left innominate vein $^{3}$ (Figure, lower panel).

The authors noted that "a PLSVC is usually asymptomatic." However, the risk of other congenital anomalies increase if a PLSVC drains into the left atrium or if the RSVC is absent. ${ }^{2,4}$ As the Figure shows, when the RSVC is absent, drainage from the right jugular and subclavian veins passes via the innominate vein into the PLSVC, and passage of a central catheter via the right jugular or the subclavian vein will appear to take an atypical course on a chest radiograph (Figure, lower panel).

With regard to the development of a PLSVC (Figure, upper panel), venous drainage of the upper embryo occurs along the right and left anterior cardinal veins. These veins drain via the right and left common cardinal veins into the

A. Verniquet, MD $(\bowtie) \cdot$ R. Kakel, MD

James Paton Memorial Hospital - Central Health,

Gander, NL, Canada

e-mail: andrewverniquet@hotmail.com sinus venosus and, finally, the primitive atrium. By the eighth week of embryonic life, a connecting vessel has developed between the right and left anterior cardinal veins, directing flow from the left to the right side. ${ }^{4}$ This connecting vein is destined to become the left innominate vein. Below the connecting vein, the left anterior cardinal vein atrophies into a fibrous cord (becoming the ligament of the left vena cava and extending from the upper part of the left superior intercostal vein to the oblique vein of the left atrium). The oblique vein of the left atrium (a remnant of the left common cardinal vein) drains into the coronary sinus (developed from the left lateral horn of the sinus venosus). Inadequate development or absence of the connecting vein leads to the development of a PLSVC, which normally drains into the right atrium via the oblique vein of the left atrium and the coronary sinus (Figure).

In conclusion, embryonic variations in venous anatomy must always be considered when performing central venous cannulation.

Competing interests None declared.

\section{References}

1. Commandeur D, Garetier M, Giacardi C, et al. Ultrasound-guided cannulation of the left subclavian vein in a case of persistent left superior vena cava. Can J Anesth 2011; 58: 471-2.

2. Goodwin JD, Chen JT. Thoracic venous anatomy. AJR Am J Roentgenol 1986; 147: 674-84.

3. Schummer W, Schummer C, Frober R. Persistent Left Superior Vena Cava and central venous catheter position :clinical impact illustrated by four cases. Surg Radiol Anat 2003; 25: 315-21.

4. Marcu C, Beek AM, van Rossum A. Unusual variation in upperbody venous anatomy found with cardiovascular MRI. CMAJ 2006; 175: 27. 

thoracic venous anatomy, from the embryo (left upper panel) to the normal adult (right upper panel). The right anterior cardinal vein evolves to become the (right) superior vena cava, and the left anterior cardinal vein involutes into a ligament of the vena cava (1"). The left anterior cardinal vein may, however, remain to become a persistent left superior vena cava that may (b) or may not (c) be connected eventually to the right superior vena cava. Rarely, the right superior vena cava is absent (a)
Figure Development of

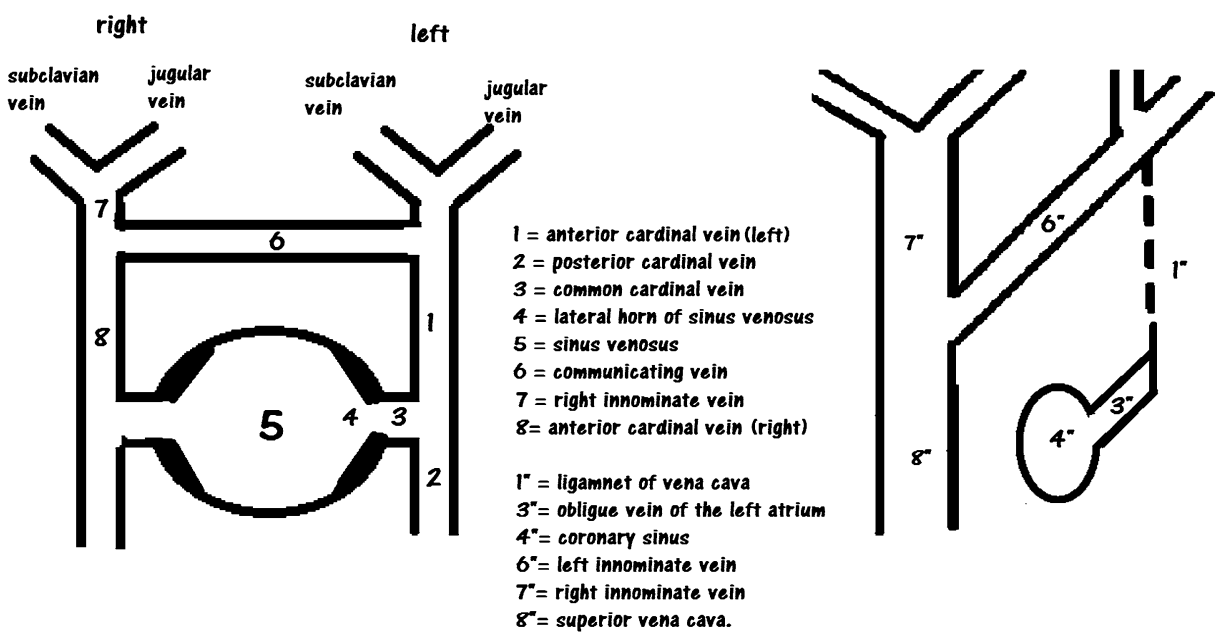

Three variations of persistant left superior vena cava

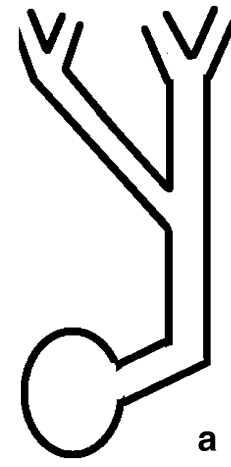

\section{...with absent right superior vena cava (rarest form)}

\section{Reply,}

We thank Drs. Verniquet et al. for their interest in our article "Ultrasound-guided cannulation of the left subclavian vein in a case of persistent left superior vena cava (PLSCV)." 1 They provide a sound description and fine illustration of embryological development of a PLSCV. We too have described this embryological development previously. ${ }^{2}$ The aim of our most recent article was to inform intensivists and anesthesiologists about an anatomical entity that could result in difficult central venous cannulation (CVC) and related diagnostic dilemmas.

Indeed, the PLSVC is a relatively common anomaly, present in $0.5 \%$ of the normal adult population and in $5-10 \%$ of patients with congenital cardiac anomalies. ${ }^{2}$ In

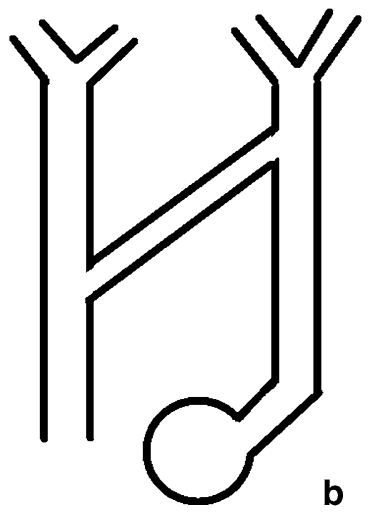

right SVC is connected to PLSVC

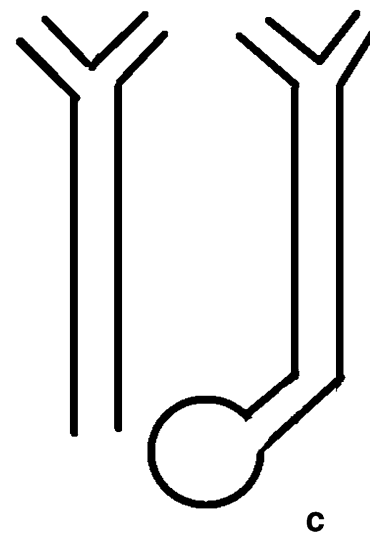

right SVC is not connected to PLSVC the absence of other cardiac malformations, the PLSCV does not in itself produce any physiological disorder. Uneventful insertion of a central venous catheter (CVC) in a PLSCV has been described by several authors. ${ }^{1,3,4}$ However, advancement of the CVC in the coronary sinus through the PLSCV has the potential to induce arrhythmias, hypotension, and angina. Perforation of the heart and tamponade can also result from guidewire insertion. ${ }^{3}$ It is for these reasons that we defined PLSCV as being "usually asymptomatic."

Diagnosing PLSCV can be difficult in the absence of other cardiac anomalies. The physical examination is unremarkable. Chest radiography usually aids in the diagnosis by showing a left-sided paramediastinal course of the CVC. The diagnosis can be confirmed by transthoracic or transesophageal echocardiography or by thoracic computed 
tomography. In the case we presented, ${ }^{1}$ false tracking of the CVC in a PLSCV was diagnosed by computed tomography of the chest. Thus, we were able to exclude a false course of the CVC in the left pericardiophrenic vein. Thoracic computed tomography is an interesting imaging modality for delineating central venous anatomy.

Competing interest None declared.

\section{References}

1. Commandeur D, Garetier M, Giacardi C, et al. Ultrasound-guided cannulation of the left subclavian vein in a case of persistent left superior vena cava. Can J Anesth 2011; 58: 471-2.
2. Ould-Ahmed M, Mas B, Hautbois E, Garcia JF, Caroff P, Guiavarch $M$. Unusual course of a pulmonary artery catheter through a persistent superior vena cava (French). Ann Fr Anesth Reanim 2000; 19: 745-8.

3. Azocar RJ, Narang P, Talmor D, Lisbon A, Kaynar AM. Persistent left superior vena cava identified after cannulation of the right subclavian vein. Anesth Analg 2002; 95: 305-7.

4. Bordes $J$, Asencio $Y$, d'Arranda E, Goutorbe P. Persistent left vena cava incidentally recognized during subclavian vein catheterization. Crit Care 2010; 14: 405.

Diane Commandeur MD

Marie-Laure Buguet-Brown MD

Mehdi Ould-Ahmed MD

Military Teaching Hospital "Clermont-Tonnerre", Brest, France 